\title{
Revista de la CEPAL
}

\author{
Director \\ RAUL PREBISCH \\ Secretario Técrico \\ ADOLFO GURRIERI \\ Secretario Adjumto \\ GREGORIO WEINBERG
}

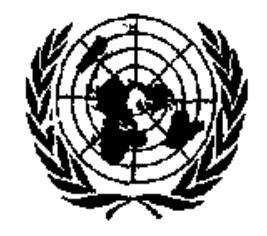

NACIONES UNIDAS

COMISION ECONOMICA PARA AMERICA LATINA

SANTIAGO DE CHILE/AGOSTO DE 1983 


\section{Revista de la \\ C E P A L}

Santiago de Chile

Número 20

\section{SUMARIO}

Nota de la Dirección

Carta del Presidente Constitucional de la República del Ecuador Don Osvaldo Hurtado a los señores Enrique V. Iglesias, Secretario Ejecutivo de la CEPAL y Carlos Alzamora, Secretario Permanente del SELA

Carta de los señores Enrique V. Iglesias, Secretario Ejecutivo de la CEPAL y Carlos Alzamora, Secretario Permanente del SELA al Presidente Constitucional del Ecuador Don Osvaldo Hurtado

Bases para una respuesta de América Latina a la crisis económica internacional. Enrique V. Iglesias y Carlos Alzamora Traverso

La crisis mundial y América Latina. Conclusiones de la Reunión de Personalidades convocada por la CEPAL y celebrada en Bogotá los dias 19 a 21 de mayo de 1983

La crisis del capitalismo y el comercio internacional. Raitl Probisch

América Latina: crisis, cooperación y desarrollo. Guillemo Maldonado, Eduar̃do Gana y Armando Di Filippo

Renegociación de la deuda latinoamericana: Un análisis del poder monopólico de la banca. Robert Devlin

Salaricis y empleos en coyunturas recesivas internacionales. Experiencias latinoamericanas recientes. Victor $\boldsymbol{E}$. Tokman

Existe una salida equitativa y democrática para laccisis: Adnffo (iurrieri y Pedro Săinz

La deuda externa y los problemas financierós de América Latina. Carlos Massad 


\section{REVISTA DE LA CEPAL N 20}

\section{Salarios y empleo en coyunturas recesivas internacionales}

\section{Experiencias latinoamericanas recientes}

\section{Víctor E. Tokman*}

E] prenemte articuko possula como hipoitesis que la recesion intesnacionat obliga a electuar ajjustes internos que implican un meomor nivel de actividad, pero que al mismo tiemus exixtell diversis onxinnes parr enlientar: la situacioni opcimes en parte determinadas por: las caracieristicas estructurater de las econnmías, pero que diependen iclemás del conjumbs cle politicus aplicadas. Paja coryoborat la hipotesis se analizan lan politicas de ajuste seguiclas por diversos parkes latimoanericanrss dusante las dox óltimas crisis, concluyendos que. si se deja de laclo al grupo de paises que porr su reclucido tamano y etevacho arato de aperimra carecen ate

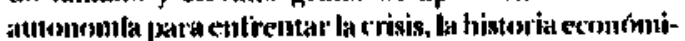
ca def ullimo quinquenios registra el tracass de las politicas monelarias gkslyales seguidas ell el ('amo Sur y ale bas polliticas cxpansivas de ks paines exportadones

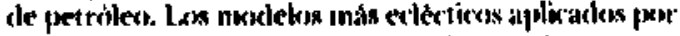
otros paises, aunque con marcadas diferencias entre sí, parecen haber sida mís efectivos para disminuir, 0 al menos postergar, el costo del ajuste y diatribuirto más equitativamente.

Además se allalizan la repercusión del aumenIt) de la lasu de inleték en las ecronomian latincamericatas

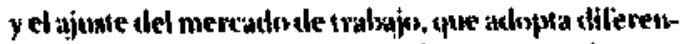

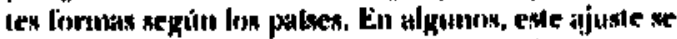

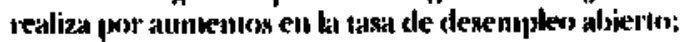

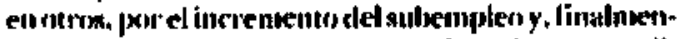

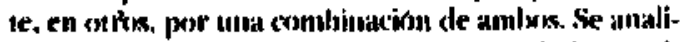
ran las causas de que en algunns paises el ajuone aclquier'a una, otra, o annless fin'mus y tambien se examiutan las implicaciones cled miknto.

"Director del Programa Regional de Empleo para America Lanina y el Caribe (PREALC).

\section{I \\ Introducción}

América Latina y el Caribe, como las demás regiones del mundo, han atravesado durante la década pasada sucesivas crisis internacionales que les han obligado a introducir políticas de ajuste en sus economias. Dicho ajuste es, sin duda, inevitable, ya que implica que las economías nacionales deben adecuarse a un menor nivel de recursos reales disponibles. Sin embargo, no hay politicas de ajuste únicas y su diversidad unida a las distintas modalidades de aplicación pueden generar resultados diferentes.

Existen diferencias, en primer lugar, en cuanto al tiempo requerido para producir los efectos deseados, entre las politicas que influyen en el nivel del gasto y las que operan, a través del cambio en los precios relativos, como mecanismo inducidor de transferencia de recursos entre sectores de producción nacional y de producción de bienes comercializables. Por otro lado, la reducción del gasto interno debería concentrarse por lo general en los niveles de consumo más que en los de inversión, para evitar posibles efectos alteradores del crecimiento a largo plazo. Asimismo, el ajuste ante la recesión internacional coincide, en la mayoría de los paises de la región, con situaciones inflacionarias, lo que obliga a plancear ambos ajustes de manera simultánea. En detinitiva, el ajuste resulta no ser neutro y recae fundamentalmente sobre los salarios y el nivel de empleo. Sobre los salarios, porque fueron considerados una variable clave tanto para la política de estabilización como para inducir aumentos en la competitividad internacional, y también para provocar reducciones en los niveles de consumo. Sobre el empleo, porque como consecuencia de la reducción del gasto interno se generan mayores niveles de desocupación abierta.

Las crisis internacionales experimentadas en la actualidad y al comienzo de la década pasada se diferencian de las fluctuaciones internacionales a las que historicamente estuvieron sometidas las economías de la región y el resto del mundo en desarrollo. De hecho, la magnitud y profundidad de dichas crisis no sólo afectian a estos países sino también a los paises centrales. En este sentido, se comparan con frecuencia creciente las coyunturas recesivas mundiales con la que alectó al mundo durante los años treinta. 
Al menos dos diferencias importantés distinguen las crisis más recientes de la producida en aquellos años. En primer lugar, dado el papel crucial que ha desempeñado en la actual crisis el alza de los precios del petróleo, el hecho de ser o no ser productor o exportador de ese combustible introduce una primera diferenciación de importancia entre los países del mundo, con independencia de que estén ubicados en el norte o en el sur. En América Latina esta diferenciación se aprecia en el comportamiento disímil que observan ante la crisis los países exportadores de petróleo con relación al resto de los países de la región. En segundo lugar, la crisis internacional actual y la anterior se plantean en medio de situaciones inflacionarias en los países desarrollados, to que tiene al menos dos implicaciones directas.

Por un lado, el deterioro de los términos de intercambio de los países no exportadores de petróleo de América Latina es incluso mayor que el registrado en ellos durante la crisis de los años treinta. Así, los términos de intercambjo de los paises latinoamericanos decrecieron a tasas anuales de 6.7 por ciento entre 1930 y 1933 , mientras que el deterioro alcanzó tasas anuales de 8.4 por ciento en la crisis de $1974-1975$ y se comprueban reducciones anuales de 9.6 por ciento desde 1978 hasta 1981 en el grupo de países no exportadores de petróleo. Sin embargo, también a diferencia de lo ocurrido en los años treinta, la contracción en el poder de compra de las exportaciones fue menor, e incluso durante la crisis actual la expansión del quantum exportado compensa con creces la caída de los términos de intercambio.

Por otro lado, las políticas seguidas en los países centrales, y en particular en los Estados Unidos, durante la recesion actual han implicado una elevación considerable de las tasas de interés nominal, lo que se produce cuando el endeudamiento de los países de la región ha crecido de manera significativa. Los incrementos de esas tasas superan el de los precios de los productos de exportación de los paises latinoamericanos no exportadores de petróleo, lo que significa por ejemplo, aumentos entre 1978 y 1981 del orden del 47.5 por ciento en la tasa prime y del orden del 32.6 por ciento en la tasa aplicable a los eurodólares, ambas en términos reales.

En este trabajo se intenta revisar la relación entre las mencionadas variables que sintetizan el efecto de la recesión internacional en la economía de los diversos países de la región y el comportamiento seguido por los salarios y el empleo. Esto se hará prestando especial atención a los períodos transcurridos entre 1973 y 1975 y desde 1979 hasta la fecha. A través de este examen podrán analizarse las diferentes respuestas dadas por los diversos países latinoamericanos ante la situación internacional y cómo las mismas afectaron a los salarios y al empleo. Para ello, y habida cuenta de la complejidad que implicaría el análisis de todos los países de la región, se intentará una tipologia aproximada de situaciones, ejemplificando el análisis con algunos casos particulares. $\mathrm{El}$ análisis, por otro lado, debe ser necesariamente parcial, ya que un enfoque comprehensivo del ajuste, por su carácter macroeconómico, supondría exceder los límites aquí propuestos.

Como hipótesis de trabajo se postula que si bien la recesión internacional obliga a efectuar ajustes internos que implican un menor nivel de actividad, existen diversas opciones para enfrentar la situación, dado que en la práctica ésta ya fue experimentada por los países de la región. Dichas opciones en parte están determinadas por las características estructurales de las economías, pero, además, dependen del conjunto de politicas que se aplique. En este contexto, la política de salarios pasa a desempeñar un papel importante en el ajuste a corto plazo $y$, en definitiva, determina la distribución del costo de dicho ajuste entre los distintos grupos de la población.

Dos aspectos adicionales serán aquí analizados. El primero, es la repercusión del alza de la tasa de interés en las economias latinoamericanas, puesto que éste es un factor nuevo que diferencia la presente crisis de las anteriores. El segundo, es el ajuste del mercado de trabajo dadas las consecuencias que tiene en los niveles y distribución del ingreso. 


\section{II \\ Los salarios y el ajuste externo}

\section{El caso de los paises pequeños y abiertos}

En primer lugar, hay en la región un grupo de países de tamaño pequeño cuyo coeficiente de apertura fluctúa entre el 20 y 30 por ciento, medido éste por la relación entre las exportaciones y el producto nacional bruto. Este grupo de paises presentaba las mismas características estructurales ya en los años treinta, y ante aquella coyuntura internacional debieron seguir ligados al patrón oro en espera de que funcionara el ajuste automático. En ese momento tuvieron que enfrentar un costo considerable dada su incapacidad para diseñar políticas que attenuaran el efecto de la crisis. En esencia, la situación actual parece ser la misma. Las crisis internacionales afectan con toda su gravedad las economías de estos países, los que no sólo deben necesariamente absorber la caída de los términos del intercambio, sino también reducir los salarios reales cada vez que se repiten esas situaciones internacionales.

Las economias centroamericanas y del Caribe son ejemplos de este tipo de países con reducidas posibilidades de autonomia. En particular, si se observan los comportamientos de Honduras y Costa Rica en la coyuntura registrada entre 1973 y 1975 y en la que se presenta desde 1979 , se advierte que en ambos países la caída en los términos de intercambio implicó también reducciones de los salarios reales. Así, en Honduras, la primera recesión representó una pérdida de crecimiento del producto, por efecto del deterioro de los términos de intercambio, del orden del 6.3 por ciento y provocó una disminución de los salarios reales del sector industrial del orden del 2.9 por ciento. La recesión actual representa hasta 1981 una desaceleración del crecimients del producto, por el mismo efecto, del orden del 8.2 por ciento, junto a una contracción de los salarios reales industriales de $\mathbf{8 . 1}$ por ciento. (Véase el cuadro 1.)

En Costa Rica, como ya se señaló, también se registran signos similares entre el efecto negativo del empeoramiento de los términos de intercambio en las dos coyunturas internacionales y la reducción de los salarios reales. En este país se suma, además, la mayor caída de los salarios reáles originada por la aceleración inflacionaria en una economía que no habia experimentado históricamente elevadas tasas de inflación. La recesión de 1973-1975 coincide allí con una inflación del orden del 25 por ciento, mientras que en la coyuntura actual la inflación entre 1979 y 1981 llega al 30 por ciento, con un registro en el último año de una tasa del 65 por ciento.

\section{El caso de los paises medianos $y$ grandes}

Ya durante la crisis de los años treinta, y en mayor medida durante las crisis internacionales más recientes, los demás países de la región mostraron poseer un cierto grado de autonomía que les permite, en alguna medida, alterar y manejar el efecto que producen las recesiones internacionales de esta magnitud. Integran este grupo de países los de mayor tamaño, por lo general menos abiertos al comercio internacional y con un grado más alto de urbanización y de modernización. Así, los coeficientes de exportación respecto al producto difícilmente superan el diez por ciento; ta proporción del empleo en las actividades urbanas modernas representa entre el $40 \mathrm{y}$ el 60 por ciento de la fuerza de trabajo total; $y$ la ocupación en la industria moderna constituye entre el 20 y el 28 por ciento de la fuerza de trabajo no agricola. Es precisamente en este grupo de paises donde el salario se convierte en una variable representativa, dada su cobertura que es función directa del grado de modernización de las economias. Este grupo incluye, entre otros, los países del Cono Sur (Argentina, Chile y Uruguay), Brasil, Colombia, México y Venezuela.

Conviene, a manera de introducción, revisar el comportamiento de estos países durante la crisis de los años treinta y las politicas seguidas por los mismos.' En primer lugar, las países lati-

'El análisis del com(montamiento de estos países y de las políticas que aplicaron durante la crisis cle kos años treinta sixue de cerca el trabajo de C. Diaz-Alejandro, Lation Amerios in the 1930s, New Haven, Economic Growth (Enter. Universidad de Yale, $19 \$ 1$. 
Cuadro I

\section{AJUSTE EXTERNO, SALARIOS Y PRECIOS EN ALGUNOS PAISES LATINOAMERICANOS}

(Poncentajes)

\begin{tabular}{|c|c|c|c|c|c|c|c|c|}
\hline & \multirow{2}{*}{$\begin{array}{l}\text { Terminos } \\
\text { de inter- } \\
\text { cambio }\end{array}$} & \multirow{2}{*}{$\begin{array}{c}\text { Grado } \\
\text { de } \\
\text { aperturab }\end{array}$} & \multirow{2}{*}{$\begin{array}{c}\text { Efecto } \\
\text { en el } \\
\text { productor }\end{array}$} & \multirow{2}{*}{$\begin{array}{c}\text { Poder de } \\
\text { compra de } \\
\text { las expor- } \\
\text { taciones" }\end{array}$} & \multicolumn{3}{|c|}{ Salariost } & \multirow[b]{2}{*}{$\begin{array}{c}\text { Precios } \\
\text { internos" }\end{array}$} \\
\hline & & & & & $\begin{array}{l}\text { Minimos } \\
\text { urbanos }\end{array}$ & $\begin{array}{l}\text { Indus- } \\
\text { triales }\end{array}$ & $\begin{array}{c}\text { Construc- } \\
\text { ción }\end{array}$ & \\
\hline $\begin{array}{l}\text { Paises pequeñ } \\
\text { Costa Rica } \\
1973-1975 \\
1975-1978 \\
1978-1981 \\
1973-1981\end{array}$ & $\begin{array}{l}\text { abiertos } \\
\begin{array}{r}9.2 \\
-\quad 40.1 \\
-\quad 99.2 \\
-\quad 9.8\end{array}\end{array}$ & $\begin{array}{l}27.6 \\
21.2 \\
21.8 \\
23.5\end{array}$ & $\begin{array}{r}-2.5 \\
8.5 \\
-6.4 \\
-\quad 2.3\end{array}$ & $\begin{array}{r}7.1 \\
-\quad 55.2 \\
-\quad 10.5 \\
29.7\end{array}$ & $\begin{array}{r}7.4 \\
25.3 \\
-\quad 8.3 \\
6.9\end{array}$ & $\begin{array}{r}9.0 \\
31.3 \\
-12.2 \\
4.9\end{array}$ & $\begin{array}{r}-6.1 \\
34.9 \\
-11.2 \\
12.7\end{array}$ & $\begin{array}{r}25.4 \\
5.9 \\
30.1 \\
19.3\end{array}$ \\
\hline $\begin{array}{l}\text { Honduras } \\
1973-1975 \\
1975-1978 \\
1978-1981 \\
1973-1981\end{array}$ & $\begin{array}{r}23.1 \\
23.3 \\
-23.7 \\
-27.6\end{array}$ & $\begin{array}{l}27.1 \\
24.1 \\
27.1 \\
26.1\end{array}$ & $\begin{array}{r}-6.3 \\
5.6 \\
-\quad 8.2 \\
-7.2\end{array}$ & $\begin{array}{r}20.2 \\
60.5 \\
-\quad 1.0 \\
32.2\end{array}$ & $\begin{array}{rr}- & 7.2 \\
- & 15.6 \\
-\quad 6.2 \\
-\quad 26.5\end{array}$ & $\begin{array}{r}2.9 \\
12.0 \\
-\quad 8.1 \\
-\end{array}$ & $\begin{array}{r}-31.9 \\
77.5 \\
-24.4 \\
-\quad 8.6\end{array}$ & $\begin{array}{r}10.4 \\
6.2 \\
14.3 \\
10.2\end{array}$ \\
\hline $\begin{array}{l}\text { Patses median } \\
\text { Dependiente } \\
\text { Brasil } \\
1973-1975 \\
1975-1978 \\
1978-1981 \\
1979-1981\end{array}$ & $\begin{array}{l}\text { ggrandes } \\
\text { Petróleo } \\
\begin{array}{r}21.2 \\
2.6 \\
-\quad 42.7 \\
-\quad 53.7\end{array}\end{array}$ & $\begin{array}{l}6.9 \\
6.2 \\
7.0 \\
6.5\end{array}$ & $\begin{array}{r}-1.5 \\
0.2 \\
-3.0 \\
-3.5\end{array}$ & $\begin{array}{r}14.7 \\
24.7 \\
6.3 \\
16.0\end{array}$ & $\begin{array}{r}2.3 \\
-3.5 \\
1.1 \\
2.3\end{array}$ & $\begin{array}{r}12.1 \\
13.3 \\
8.1 \\
97.3\end{array}$ & $\begin{array}{r}6.9 \\
12.0 \\
-\quad 1.9 \\
17.4\end{array}$ & $\begin{array}{l}32.5 \\
42.0 \\
87.3 \\
54.8\end{array}$ \\
\hline $\begin{array}{l}\text { Con mayor } \\
\text { autoabasteci } \\
\text { Con cambios } \\
\text { politicas eco } \\
\text { Argentina } \\
1979-1975 \\
1975-1978 \\
1978-1981 \\
1973-1981\end{array}$ & $\begin{array}{l}\text { lo de } \\
\text { nto } \\
\text { ofundos de } \\
\text { iicas } \\
\begin{array}{rr}-\quad 30.0 \\
-\quad 19.2 \\
-. & 8.4 \\
- & 48.2\end{array}\end{array}$ & $\begin{array}{l}5.5 \\
9.2 \\
8.1 \\
7.5\end{array}$ & $\begin{array}{l}-1.7 \\
-1.8 \\
=0.7 \\
-3.6\end{array}$ & $\begin{array}{r}45.9 \\
87.2 \\
-\quad 3.3 \\
24.0\end{array}$ & $\begin{array}{r}8.9 \\
-\quad 50.4 \\
6.3 \\
-\quad 52.0\end{array}$ & $\begin{array}{r}1.7 \\
-54.9 \\
14.7 \\
-20.6\end{array}$ & $\begin{array}{r}33.3 \\
-55.9 \\
-3.2 \\
-43.1\end{array}$ & $\begin{array}{l}173.8 \\
211.5 \\
118.2 \\
163.9\end{array}$ \\
\hline $\begin{array}{l}\text { Chile } \\
1970-1975 \\
1975-1978 \\
1978-1981 \\
1970-1981\end{array}$ & $\begin{array}{rr}- & 46.8 \\
- & 0.6 \\
- & 14.0 \\
- & 57.2\end{array}$ & $\begin{array}{l}15.3 \\
21.2 \\
24.6 \\
210.4\end{array}$ & $\begin{array}{l}-7.2 \\
-0.1 \\
-3.4 \\
-11.7\end{array}$ & $\begin{array}{r}28.7 \\
27.1 \\
13.7 \\
8.19\end{array}$ & $\begin{array}{r}-41.1 \\
29.9 \\
-\quad 1.5 \\
-\quad 24.7\end{array}$ & $\begin{array}{r}-41.8 \\
44.3 \\
38.0 \\
15.9\end{array}$ & $\begin{array}{r}-18.3 \\
4.2 \\
27.0 \\
8.1\end{array}$ & $\begin{array}{r}233.1 \\
80.1 \\
25.9 \\
98.9\end{array}$ \\
\hline $\begin{array}{l}\text { Sin cambios } \\
\text { politicas eco } \\
\text { ('olombja } \\
1973-1975 \\
1975-1978 \\
1978-1981 \\
1973-1981\end{array}$ & $\begin{array}{l}\text { fundos de } \\
\text { icas } \\
\begin{array}{r}19.9 \\
64.7 \\
-\quad 32.4 \\
-\quad 7.4\end{array}\end{array}$ & $\begin{array}{r}14.8 \\
7.4 \\
6.9 \\
9.7\end{array}$ & $\begin{array}{r}-2.9 \\
4.8 \\
-\quad 2.2 \\
-\quad 0.7\end{array}$ & $\begin{array}{r}0.4 \\
51.7 \\
-\quad 23.8 \\
16.1\end{array}$ & $\begin{array}{r}16.4 \\
9.4 \\
17.6 \\
49.7\end{array}$ & $\begin{array}{r}8.1) \\
13.3 \\
7.2 \\
11.7\end{array}$ & $\begin{array}{r}7.3 \\
5.1 \\
17.6 \\
92.6\end{array}$ & $\begin{array}{l}22.3 \\
24.2 \\
27.7 \\
25.0\end{array}$ \\
\hline $\begin{array}{l}\text { Patises export } \\
\text { petroleo } \\
\text { Ecuador } \\
1973-1975 \\
1975-1978 \\
1978-1981 \\
1973-198 \text { I }\end{array}$ & $\begin{array}{r}36.3 \\
8.7 \\
31.1 \\
94.3\end{array}$ & $\begin{array}{l}18.1 \\
12.4 \\
11.4 \\
14.0\end{array}$ & $\begin{array}{r}6.6 \\
1.1 \\
3.6 \\
13.2\end{array}$ & $\begin{array}{l}16.5 \\
27.7 \\
28.1 \\
96.0\end{array}$ & $\begin{array}{r}4.3 \\
-\quad 8.0 \\
111.3 \\
112.5\end{array}$ & $\begin{array}{r}7.3 \\
8.4 \\
22.9 \\
43.0\end{array}$ & $\begin{array}{l}\cdots \\
\cdots \\
\cdots \\
\cdots\end{array}$ & $\begin{array}{l}17.1 \\
11.6 \\
13.7 \\
13.7\end{array}$ \\
\hline
\end{tabular}


Continuación del cuadro 1.

\begin{tabular}{|c|c|c|c|c|c|c|c|c|}
\hline & \multirow{2}{*}{$\begin{array}{l}\text { Términos } \\
\text { de inter- } \\
\text { cambio* }\end{array}$} & \multirow{2}{*}{$\begin{array}{c}\text { Grado } \\
\text { de } \\
\text { aperturab }\end{array}$} & \multirow{2}{*}{$\begin{array}{c}\text { Efecto } \\
\text { en ef } \\
\text { productor }\end{array}$} & \multirow{2}{*}{$\begin{array}{l}\text { Poder de } \\
\text { compra de } \\
\text { las expor- } \\
\text { tuciones" }\end{array}$} & \multicolumn{3}{|c|}{ Salarios" } & \multirow[b]{2}{*}{$\begin{array}{l}\text { Precios } \\
\text { internos" }\end{array}$} \\
\hline & & & & & $\begin{array}{l}\text { Minimos } \\
\text { urbanos }\end{array}$ & $\begin{array}{l}\text { Indus- } \\
\text { triales }\end{array}$ & $\begin{array}{l}\text { Construc- } \\
\text { ción }\end{array}$ & \\
\hline $\begin{array}{l}\text { México } \\
1973-1975 \\
1975-1978 \\
1978-1981 \\
1973-1981\end{array}$ & $\begin{array}{r}3.6 \\
12.2 \\
47.6 \\
71.5\end{array}$ & $\begin{array}{l}7.3 \\
7.0 \\
7.4 \\
7.2\end{array}$ & $\begin{array}{l}0.3 \\
0.8 \\
3.5 \\
5.1\end{array}$ & $\begin{array}{r}2.5 \\
82.3 \\
140.6 \\
349.8\end{array}$ & $\begin{array}{r}16.9 \\
7.9 \\
-\quad 8.4 \\
15.6\end{array}$ & $\begin{array}{r}8.6 \\
8.0 \\
-\quad 1.7 \\
14.0\end{array}$ & $\begin{array}{r}5.8 \\
9.1 \\
1.6 \\
17.3\end{array}$ & $\begin{array}{l}15.8 \\
21.3 \\
26.1 \\
21.7\end{array}$ \\
\hline $\begin{array}{l}\text { Venezuela } \\
1973-1975 \\
1975-1978 \\
1978-1981 \\
1973-1981\end{array}$ & $\begin{array}{r}97.6 \\
-13.9 \\
114.2 \\
264.4\end{array}$ & $\begin{array}{r}14.3 \\
9.5 \\
8.1 \\
10.6\end{array}$ & $\begin{array}{r}14.0 \\
-\quad 1.3 \\
9.3 \\
28.0\end{array}$ & $\begin{array}{r}30.3 \\
-\quad 20.4 \\
77.2 \\
102.3\end{array}$ & $\begin{array}{r}-9.3 \\
-\quad 19.6 \\
-17.7 \\
-\quad 14.2\end{array}$ & $\begin{array}{r}13.1 \\
2.1 \\
-\quad 1.1 \\
14.2\end{array}$ & $\begin{array}{c}\ldots \\
-\quad \ldots \\
\ldots\end{array}$ & $\begin{array}{r}9.8 \\
7.3 \\
17.8 \\
11.8\end{array}$ \\
\hline
\end{tabular}

Fuenir: Elaboración del PREALC a base de datos nacionales.

* Vatiaciones entre los años inicial y final de cada subperikado.

1. Relación entre exportaciones y productos interno bruto.

- Se ubuvo multiplicando las variaciones en los términos de intercambio por al coetiviente de apertura.

- Tasas acumulativas aruales referidas a las variaciones de diciembre a diciembre.

noamericanos con cierto grado de autonomía atraviesan esa crisis con un costo menor que los demás paises de la región, y aun que Estados Unidos y Canadá. En general, crecen más rápidamente que estos dos países en el periodo posterior a la crisis (1932-1939); se recuperan antes; su contracción no es tan acentuada como en los paises de América del Norte; y la industria manufacturera, que se convierte en el sector líder, crece según los casos entre el 13 y el 8 por ciento al año de manera sostenida, mientras que en kss Estados Unidos la industria manufacturera se estanca.

Las políticas seguidas por estos paises durante la crisis de los años treinta se caracterizan por la heterodoxia, que desafia a las recetas convencionales de los expertos monetaristas de la epoca (momey doctors, según Carlos Díaz-Alejandro). Con relación al balance de pagos, devalúan sus monedas y establecen un sistemat de cambio múltiple: un tipo de cambro más elevado para las importaciones, uno intermedio para las exportaciones, y un tipo de cambio preferencial para el pago de la deuda externa. Asimismo, la mayoría de ellos renegocia unilateralmente la deuda; $y$ sólo Argentina, dentro de este grupo de países, continúa payando el servicio de la misma. El efecto de la devaluación no resultó inflacionario y contribuyó a aumentar la competitividad al modificar la relación entre tipo de cambio y satlarios, fundamentalmente por el reajuste del primero ya que los segundos se mantuvieron estables en términos reates. $\Lambda$ las nedidas cambjarias se sumaron controles de canıbio y ajustes de aranceles que elevaron el grado de protección.

Las políticas monetaria y fiscal entonces seguidas se caracterizan por su efecto expansivo. Se incrementa la oferta monetaria real, sobre todo por la calda de los precios, y se emite de manera independiente contra bonos que no guardan relación con el patrón oro vigente hasta entonces. Varios de estos países declaran una moratoria de la deuda interna y para evitar su quiebra se apoya al sistema financiero con diversas medidas que van desde la apertura de líneas de redescuento especial hasta, en algunos casos, la congelación de los depósitos de ahorro. La política fiscal adquiere características anticíclicas que no tratan de equilibrar el presupuesto y mantienen un déficit que se financia básicamente con retrasos en los pagos y se acude a la deuda flotante. Asimismo, el gasto público se incrementa para evitar una reducción de la actividad económica.

Por último, la combinación de las medidas adoptadas tiene como resultado un profundo cambio estructural, el que caracteriza hasta el presente a las economías de estos países y consiste en una acelerada expansión de la industria manufacturera y en una mayor participación del Estado en la actividad económica.

En la actualidad, los países de este grupo 
comienzan a diferenciarse entre si debido esencialmente a la repercusión que tienen los precios del petróleo en las últimas crisis internacionales. Ello hace necesario distinguir los países exportadores de petróleo de los que dependen sustancialmente de combustible importado y de aquellos otros que poseen cierto grado de autonomía, dado su alto grado de autoabastecimiento. Por una parte, dentro del grupo de países exportadores de petróleo están Ecuador, México y Venezuela, entre otros. Por otra, entre los que dependen de manera significativa de los abastecimientos importados de combustibles está el caso particular de Brasil. Por último, dentro del grupo de paises con un mayor grado de autoabastecimiento, cabe distinguir a aquellos que durante la década pasada aplicaron políticas estructurales de estabilización y apertura (fundamentalmente, los del Cono Sur) de aquellos que siguieron políticas más convencionales, como sería el caso de Colombia.

\section{Los paises exportadores de petroleo}

Estos paísés se vieron beneficiados últimamente por el alza del precio del petróleo, lo que determinó un aumento significativo de sus términos de intercambio, combinado en algunos casos, como el de México, con una expansión muy considerable del volumen exportado de ese producto. Se consideran en particular dentro de este grupo a Ecuador, México y Venezuela. Estos tres países pudieron seguir políticas de salarios expansivas aprovechando las favorables coyunturas internacionales y por lo tanto se advierte en ellos una estrecha correlación entre la elevación de los términos de intercambio y el aumento de los salarios reales. (Vease de nuevo cuadro 1.)

No obstante lo señalado, en dichos países se desarrolla como consecuencia del mismo auge petrolero, una tendencia a la sobreexpansión del gasto interno y al incremento de la masa monetaria, la que tiene como resultado una aceleración inflacionaria, problemas de balance de pagos o una combinación de ambos. Estos fenómenos constituyen consecuencias paradójicas en economías que históricamente no habłan registrado tasas de inflación significativas y que, además, combinan los períodos de bonanza más pronunciados de las últimas décadas con la existencia de déficit en sus balances de pagos. Ello los impulsas a recurrir a políticas de ajuste que implican reducción del gasto y control de los salarios, como ocurrió en México y Venezuela a partir de 1979. Por consiguiente, los salarios reales tienden a contraerse a pesar de la expansión de los términos de intercambio.

En definitiva, los desequilibrios internos y las políticas seguidas determinan que, si bien durante el período 1973-1981 los salarios se ven beneficiados por el auge que experimentan estos países, los beneficios transferidos disminuyen debido a la existencia de esos desequilibrios, en particular de la inflación, por tratarse de economias con escasa experiencia inflacionaria $y$, por lo tanto, con sistemas de salarios no indizados. Así, en Venezuela, frente a una expansión del producto atribuible a la mejora de los términos de intercambio, del orden del 28 por ciento, los salarios reales del sector industrial sólo se expanden en 14 por ciento durante el mismo período.

\section{Los patses no exportadores de petróleo}

\section{a) Los patises dependientes de las importaciones de petróleo}

Se cita aqui el caso particular del Brasil, país que muestra autonomía en el diseños de sus políticas económicas, pero experimenta a la vez una fuerte dependencia con respecto a las importiaciones de petróleo. Por otro lado, utiliza de manera activa las posibilidades de endeudarse en el exterior para amortiguar el efecto recesivo de la pronunciada calda de sus términos de intercambio.

Como resultado de dichas políticas, Brasil pudo, durante el período 1973-1975, incrementar los salarios industriales en términos reales en alrededor del 12 por ciento, a pesar de que sus terrminos de intercambio cayeron en alrededor del 21 por ciento. Sin embargo, frente a la nueva reducción de sus términos de intercambio, que se produce a partir de 1978, los salarios reales desaceleran significativamente su crecimiento. (Véase el cuadro 1.)

Es evidente que Brasil pierde flexibilidad en el manejo de las crisis internacionales, tanto por la presencia de desequilibrios internos que en el último período se manifiestan fundamentalmente por una intensificación de la inflación como por el mayor costo que le significa el aumento de las tasas internacionales de interés en 
términos reales, consecuencia de su alto grado de endeudamiento. De esta forma, si se compara el crecimiento de la tasa de interés nominal aplicada por los bancos comerciales a los mejores deudores (prime rate) y el de la aplicada a los depósitos a tres meses en eurodólares con el aumento de los precios de las exportaciones brasileñas, se advertirá que la primera, en el periodo 1978-1981, aumentó en 59 por ciento, y la segunda en 43 por ciento, ambas en términos reales. Dado que el 84 por ciento de la deuda externa desembolsada brasileña está contratado a tasas de interés variable, y que la misma constituye alrededor del 25 por ciento del producto nacional bruto, si se tiene en cuenta que la tasa vigente en el año 1978 era alrededor del 10 por ciento, puede estimarse que su efecto en las variaciones de la tasa de interés internacional representa un deterioro en el producto del orden del 11 por ciento, lo que equivale a alrededor de cuatro veces el deterioro del producto atribuible a la caída de los términos de intercambio en el período 1978-1981.

Sin embargo, no obstante la coyuntura desfavorable que Brasil enfrenta en la actualidad, el análisis del período 1973-1981 señala que en dicho pais, a pesar de haberse tenido que enfrentar términos de intercambio desfavorables que significan una reducción en el crecimiento del producto cercano al 3.5 por ciento, las politicas internas seguidas no sólo hicieron posible no trasladar dicha reducción a los salarios, sino por el contrario, contribuyeron a que aumentaran significativamente, en alrededor del 37 por ciento en términos reales, los salarios pagackos en el sector industrial. Fs éste, sin duda alguna, un claro casos de políticas que pernitieron enfrentar la recesión internacional sin hàcer recaer, en primera instancia, el peso del ajuste en los salarios. Es todavía prematuro evaluar el efecto neto, ya que este pais debe hacer frente a uná cuantiosa deuda externa que fue la que en parte posibilitó el manejo efectuado de la política económica.

b) Los paises no deperdientes del petróleo

i) Paises sin cambios importantes de políticas económicas. Un caso ilustrativo de este tipo de situacionè es Colombia. Los términos de intercambio del país se vieron adversamente afectados tanto durante la coyuntura $1973-1975 \mathrm{como}$ durante la recesión que comenzó en 1978. A pesar del efecto adverso registrado en la situa- ción internacional, las politiras económicas seguidas permitieron expandir el quantum de las exportaciones, neutralizando así el efecto negativo de los precios internacionales y posibilitando a su vez expandir de manera significativa los salarios reales durante el período 1973-1981. Así, ante una contracción potencial del producto, atribuible a la caída de los têrminos de intercambio, de cerca del uno por ciento, los salarios minimos urbanos se expanden en 50 por ciento, los industriales en 12 por ciento y los de la construcción en 33 por ciento, todos ellos en términos reales, durante el mismo período. (Véase de nuevo el cuadro 1.)

ii) Paises con acentuados cambios de politicas económicas. Este grupo incluye Argentina, Chile y Uruguay, ${ }^{2}$ países que durante la década siguieron políticas de estabilización y apertura de sus economías. No corresponde caracterizar aquí las politicas seguidas, ni tampoco sus efectos principales" sino que nos limitaremos a destacar algunos aspectos que se relacionan con el grado de autonomía que poseen estos países en la toma de decisiones de política económica interna y con los resultados que se registraron como consecuencia de las políticas adoptadas.

En primer lugar, el hecho de que estos paises cuentan con autonomia en sus decisiones de politica económica y no están enteramente sujetos a las fluctuaciones internacionales, lo ilustra con claridad el caso de Argentina, que durante la coyuntura de 1973 a 1975 logra, a pesar de sufrir una importante reducción en sus términos de intercambio de alrededor del 30 por ciento, que esta situación no se refleje en una caída de los salarios en términos reales. Asimismo Chile, en la coyuntura que comienza en 1978, logra evitar el traslado del deterioro de los términos de intercambio hacia la reducción de los salarios. Debe señalarse en el último caso que el aumento de los salarios reales durante el último período obedece, en parte significativa, a la desaceleración registrada en la inflación, lo que automáticamente, en economias indizadas como las de este grupo, se traduce en aumento de los salarios reales.

${ }^{2}$ Debe señalarse que los tres paises presentan distinto grado de dependencia con respecto al petróles importacls. Argeutina es prácticamente autosuficiente $y$ Uruguay nuy clependiente. (hile se sitúa en un lugar intermedio.

"Véase, por ejemplo, PREALC, Politiós de ratubitizarión y empleo en America Latina, Serie Investigaciones solbre Empleo/22, Santiago, PREALC, 1982. 
En segundo lugar, los salarios pasan a constituir una variable fundamental dentro de la politica económica y social adoptada por estos paises. El control de los salarios constituyó un instrumento privilegiado en los esfuerzos de estabilización y de incremento de la competitividad internacional, en especial durante los períodos bastante prolongados en que se siguieron políticas de tipo de cambio fijo (o, más en general, de sobrevaluación de la moneda local), cuando se buscaba, a través de la reducción de los salarios, el traslado de los recursos hacia la producción de bienes comercializables. Se utilizo asimismo el control de los salarios como un objetivo social de disciplinamiento de la fuerza de trabajo, ya que ambos paises buscaron por este medio evitar presiones que contradijesen las decisiones económicas y políticas propuestas. En particular, la estrategia económica seguida tuvo como resultado la desarticulación de la estructura sindical, fundamentalmente como consecuencia de la orientación antiindustrial y antiempleo que la misma introdujo. 'Esto permite hacer efectivas reducciones en los salarios reales sin que se generen fuerzas dentro del sistema que traten de impedirlo.

En definitiva, el efecto de estas políticas hace que la reducción de los salarios exceda el costo que significaría absorber la pérdida de los términos de intercambio. Así, en Argentina, entre 1973 y 1981 , dicho deterioro significa una disminución del crecimiento del producto del orden del 3.6 por ciento, cuando los salarios del sector industrial se contraen durante ese mismo período en alrededor del 21 por ciento. En Chile, entre 1970 y 1975 , el efecto negativo de la caída de los términos de intercambio representa una reducción del producto de alrededor del 7 por ciento, pero los salarios industriales se contraen en más de 41 por ciento. (Véase nuevamente el cuadro 1.)

Sin embargo, en ambos paises esos mismos salarios, tambièn en términos reales, aumentan entre 1978 y 1981 frente a caídas en los términos de intercambio. Este comportamiento salarial oculta por diversas razones el efecto real de las

'R. Lagos y V.E. Tokman, Monetarismo global, empleos y estratificación social, Serie Trabajos Ocasionales/47, Rev. 1 , Santiago, PREALC; 1982. políticas seguidas. La primera, es que 1981 es un año de máxima para los salarios, que no perdura; por el contrario, los salarios realés pagados en la industria se contraen durante 1982 , en 10.5 y en 3 por ciento en Argentina y Chile, respectivamente. La segunda es que el ajuste se efectuó principalmente a través de reducciones en los niveles de ocupación total, y, en particular, de la industria manufacturera, más que a través de reducciones en los salarios. Así, el empleo industrial en Argentina cae en términos absolutos entre 1973 y 1981 en 32.8 por ciento ( 37.2 por ciento si se incluye 1982) y en 21.2 por ciento entre 1978 y 1981 (26.2 por ciento si se incluye 1982). En'Chíle, la reducción entre 1970 y 1981 es de 17 por ciento (28 por ciento si se incluye 1982) y de 8 por ciento entre 1978 y 1981 (20.4 por ciento si se incluye 1982). Por último, en el caso particular de Chile, el ajuste implicó un alza sustancial de la tasa de desempleo abierto y de ocupaciones en programas de emergencia con remuneraciones de alrededor de un tercio del salario mínimo. La tasa de desocupación, incluyendo el empleo mínimo, se quintuplica entre 1970 y 1981 y llega al 28.4 por ciento de la fuerza de trabajo en 1982.

\section{Conclusiones tentativas}

Fn resumen, la experiencia de los paises latinoamericanos señala que si bien ante coyunturas internacionales adversas las ecomomias no tienelt otra alternativa que ajustarse a la situación y que dicho ajuste implicat políticas ste reducción de los salarios reales, ello sólo es aplicable de manerat autonática a un reducido grupo de paises que por su tamaño y grado de apertura no disponen de autonomia suficiente para alterar dicho resultado. Sin embargo, hay un número significativo de paises en la región que ya descle hace algunas décadas disponen de autonomía como parat orientar su djuste interno a las recesiones internacionales, influyendo en la distribución de su costo, en el período durante el cual se introdnce y, en definitiva, en los mecanismus por los cuales aclecuan sus economias a las diversas coyunturas externas. Ia experiencia muestra que algumos paises lograron aplicar políticas de silarios expansivas frente a condiciones internacionales adversas, mientras que otros acentuaron el costo del ajuste al hacer coincidir la introducción de 
cambios importantes en sus politicas económicas con esas mismas coyunturas internacionales. Por áltimo, el caso de los paises exportadores de petróleo constituye una situación particular, pues annque es en principio posible -y asi lo han hecho-traducir la bonanza internacional en aumentos de los salarios reales, surgen desequilibrios internos, $y$ a veces externos, que terminan por malograr cticha posibilidad $y$, en definitiva, los salarios crecen menos de lo que hubiera sido teóricamente factible.
En suma, la historia económica del último quinquenio registra el fracaso de dos tipos de políticas. Las políticas monetarias globales de efectos contractivos aplicadas en el Cono Sur y las politicas expansivas de los países exportadores de petróleo. Los modelos más eclécticos seguidos por otros paises, aunque con marcadas diferencias entre sí, parecen haber sido más efectivos para disminuir, $o$ al menos postergar, el costo del ajuste y distribuirlo más equitativamente.

\section{III}

\section{El problema del alza de la tasa de interés internacional}

La actual recesión internacional se diferencia de las anteriores en que no sólo se produce una caida pronunciada en los términos de intercambio de los paises de la región, sino que se registra también un alza de la tasa de interés aplicada a los pagos de la deuda externa. Así, entre 1978 y 1981 , la tasa que rige para los mejores clientes de los bancos norteamericanos (prime rate) se eleva en 72.3 por ciento, y la correspondiente a los depósitos en eurodólares a tres meses aumenta 54.9 por ciento.

Este considerable incremento de las tasas de interés nominales significa para los países de la región una salida adicional de recursos financieros. Así, se estima que en 1981, cada punto que sube la tasa de interés implica pagos adicionales por este concepto ascendentes a $2250 \mathrm{mi}-$ llones de dólares para la región en su conjunto. Dichos pagos adicionales representan a su vez, alrededor de 2,6 por ciento de las exportaciones de bienes de América Latina, para alcanzar en algunos paises, como México, a 3.3 por ciento de sus exportaciones, y en Argentina y Brasil, a aproximadamente 3 por ciento de sus ventas al exterior.

Para evaluar la presión de recursos que significa el mencionado aumentu de las tasas de interes nominales, conviene relacionarlo con la evolución de los precios de las exportaciones de los países latinoamericanos durante dicho período. Cuando se efectúa esta comparación la situa- ción tiende a diversificarse de acuerdo con la experiencia de cada país. En los paises exportadores de petróleo, que durante el período registraron un mejoramiento de sus precios de exportación mayor que el alza experimentada por la tasa de interés nominal, la variación de la tasa de interés real es, por ende, negativa. En el resto de los paises de la región, el efecto neto de la variación de la tasa de interés tiende a influir de manera desigual en las posibilidades de crecimiento del producto, según la evolución de sus precios de exportación, del porcentaje que representa la deuda externit con respecto al producto nacional bruto y de la proporción de dicha deuda sujeta a tasas de interés variable.

Se pueden distinguir, en primer lugar, aquellos países donde el alza de las tasas de interés nominales se combinó con una catida de loss precios de exportación y con una deuda externa equivalente a una elevada proporción de su producto nacional bruto. Estos son típicamente los casos de las economías centroamericanas, entre ellas Costa Rica y Honduras, donde por el almento de la tasa de interés real ${ }^{5}$ se ven disminui-

El método seguido para calcular este efecto consistió en estimar la proponcion de la deuda sujeta a casas de interes variables con relacion al producto interno bruks, y se aplicis a dicho coeticiente la tasa de crecimiento de la tasa de interés real, definida como la variación de la tasa de interés internacional deflactada por la variación de los precios de las exportaciones del país respectivo. 
das sus posibilidades de crecimiento. Así en Costa Rica la pérdida sería del orden del 20 por ciento y en Honduras del 16 por ciento, durante el período 1978-1981. (Véase el cuadro 2.) En segundo lugar, existe otro grupo de países donde el alza de la tasa de interés nominal se combina con un cuasi estancamiento de sus precios de exportación, y cuya deuda externa representa alrededor del 25 por ciento del producto, aunque está en su mayor parte sujeta a tasas de interés variables. Estos son, por ejemplo, los casos de Brasil y Culombia, en que el efecto de pérdida potencial del crecimiento del producto es de alrededor de 10 por ciento durante el mismo período. Por último, hay un grupo de paises en los cuales el crecimiento de la tasa de interés se ve en gran parte compensado con el alza de los precios de sus exportaciones, pero que a la vez se registran altos coeficientes de deuda con relación al producto, cercanos al 40 por ciento, y una proporción de aproximadamente 80 por ciento de su deuda está sujeta a tasas de interés variables. Son éstos los casos de Argentina y Chile, para los cuales se estiman pérdidas potenciales de 4 y 7 por ciento, respectivamente.
Por otro lado, para tener una idea aproximada de la magnitud del efecto de esta alza de la tasa de interés real con relación a la pérdida originada por la evolución de los términos de intercambio, conviene relacionar ambos efectos en el crecimiento potencial del producto. Resulta así que la magnitud del primer efecto señalado, durante el periodo 1978-1981, varía entre 1.9 y 5.6 veces la del segundo, según los países. En el extremo superior se encuentran los casos de Argentina y Colombia, donde el primer efecto supera en alrededor de cinco veces al segundo; les sigue un grupo intermedio, constituido рог Brasil y Costa.Rica, en que el primer efecto triplica aproximadamente al segundo; $y$, finalmente, están los casos de Chile y Honduras en los cuales el primer efecto casi duplica al segundo. (Véase de nuevo el cuadro 2.)

Conviene, por último, destacar que el cambio más importante en la estructura de la deuda externa registrado en los últimos años consiste en un aumento de la participación de los fondos privados, considerando tanto su origen como su destino. En particular, se observa un fuerte incremento de la participación de los fondos del

Cuadro 2

EFECTOS DE LA VARIACION DE LAS TASAS DE INTERES INTERNACIONALES, 1978-198:

\begin{tabular}{|c|c|c|c|c|c|c|c|c|}
\hline & \multirow{2}{*}{$\frac{i_{1}}{p_{x}}$} & \multirow{2}{*}{$\frac{i q}{p_{x}}$} & \multirow{2}{*}{$\frac{\mathrm{i}_{1}}{\mathrm{p}_{\mathrm{i} / \mathrm{t}_{\mathrm{c}}}}$} & \multirow{2}{*}{$\frac{\mathrm{i}_{2}}{\mathrm{Pi} / 1_{\mathrm{c}}}$} & \multicolumn{2}{|c|}{$\begin{array}{l}\text { Pimeritije de la } \\
\text { deudla externa }\end{array}$} & \multirow{2}{*}{$\begin{array}{l}\text { Pérodida de } \\
\text { concinniesules } \\
\text { pur alza de } \\
\text { Lasits de inte. } \\
\text { rés" }\end{array}$} & \multirow{2}{*}{$\begin{array}{l}\text { Relación pérali- } \\
\text { da de creci- } \\
\text { mientu poralza } \\
\text { de tases de in- } \\
\text { terés y por de } \\
\text { terioro terni- } \\
\text { nos de inter } \\
\text { cambiot } \\
\text { (X) }\end{array}$} \\
\hline & & & & & $\begin{array}{l}\text { con respecto al } \\
\text { producto na* } \\
\text { cional bruto" }\end{array}$ & $\begin{array}{l}\text { sujeco a latsas } \\
\text { ale jinteres vid- } \\
\text { riables's }\end{array}$ & & \\
\hline Argentina & 5.9 & 17.8 & -57.8 & -47.5 & 41.1 & $8(1.0$ & -3.9 & 5.6 \\
\hline Brasil & 42.9 & 58.9 & 26.1 & 40.9 & 24.9 & 84.0 & -10.7 & 3.6 \\
\hline Colombia & 68.1 & 86.9 & 7.2 & 19.2 & 22.8 & 58.3 & $-(0) .3$ & 4.7 \\
\hline Costa Rica & 56.0 & 73.6 & 99.4 & 125.5 & 49.3 & 62.4 & -19.9 & 3.1 \\
\hline Chile & 22.1 & 35.8 & -14.4 & -4.8 & 35.6 & 6.5 .6 & -6.8 & 2.0 \\
\hline Honduras & 49.9 & 66.7 & 8.0 & 20.2 & 59.6 & 45.8 & -15.9 & 1.9 \\
\hline
\end{tabular}

Fuente: Elaborado por PREALC: a base de intormación de los países.

Notas: is = variación de la tasa de interés sobre depósitos en curoulólates a 901 días.

i. = variación de la tasa de interés prime.

$\mathrm{F}_{\mathrm{x}}=$ cambios en los precios de las exportaciones.

$P_{i}=$ cambios en los precius intermos.

$\mathbf{c}_{\mathrm{c}}=$ cambios en el tipo de cambio.

- Se refiere a 1980 .

, Se refiere al promedio $1978-1980$.

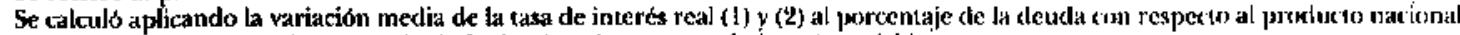
bruto (5), ajustado por el porcentaje de la deuda sujpta a tasas de interes variatbles.

1 Relación entre ( 7 ) y el efecto pérdida de los términos de iniercainbio serún cl cuadros. 1. 
sector privado sin garantía del sector público. Todo ello ha modificado las características del endeudamiento, y éste ha ido quedando sujeto en proporción creciente ( $85 \%$ hacia 1981) a tasas de interés variables; en consecuencia, se ha hecho más vulnerable a la evolución de estas tasas en los mercados financieros internacionales.

Desde el punto de vista distributivo también conviene comparar el efecto del alza de la tasa de interés nominal con la evolución de los precios significativos para los prestatarios privados nacionales. Para ellos los precios significativos están determinados por los cambios de los precios internos frente a las variaciones del tipo de cambio. Cuando se efectúa dicha comparación, se comprueba que, en general, el efecto del alza de la tasa de interés nominal no se ha visto trasladado a los prestatarios privados nacionales por cuanto la mayoría de los paises registra tipos de cambio fijos con aumento de los precios internos, o rezagos cambiarios con relación a la inflación interna.

Casos evidentes de tipo de cambio fijo con incremento de precios internos son los países exportadores de petróleo, en particular México y Venezuela, al igual que las economias pequeñas y abiertas como la de Honduras que también mantiene un tipo de cambio fijo frente a alzas de los precios internos. Ello tiene como resultado, desde el punto de vista de quienes se endeudan en dólares, una tasa de interés negativa. Siluación similar se registra en los casos de Argentina y (Chile, países que durante el periodo estudiado aplicaron politicas de estabilización que utilizan tanto el tipo de cambio tijo como rezagos cambiarios considerables como instrumentos para disminuir las presiones intlacionarias. En estos dos casos, la tasa de interés para los endeudados en dólares es igualmente negativa. Asimismo, en los casos de Brasil y Colombia, también se registran rezagos cambiarios, aunque de menor intensidad que en los paises antes mencionados, y su efecto es reducir el aumento de la tasa de interés real en alrededor de la mitad en Brasil y a la novena parte en Colombia. Por último, en otros países, como Costa Rica, el alza de la tasa de interés nominal se combina con una apreciación en términos reales del tipo de cambio, lo que tiene como resultado una tasa de interés real positivit más elevada para el deudor privado que para el país en su conjunto.

En síntesis, es evidente que el aumento de la tasa de interés nominal en tos mercados financieros internacionales registrado durante los últir mos años significa una considerable carga financiera adicional para los países latinoamericanos que deben ajustir sus economías frente a una situación recesiva como la predominante en la actualidad. No obstante, cuando se analiza el efecto del alza de la tasa de interés real, las situaciones difieren de acuerdo con la evolución de los precios de las exportaciones, el peso de la deuda con relación al producto y la proporción de la misma sujeta a tasas de interés variables. Por último, la incidencia del alza de la tasa de interés nominal en el sector privado nacional depende de la política cambiaria seguida por el país y de la evolución de los precios internos; asi, en la mayoría de los casos, se registra una carga menor que la determinada por la variación de la tasa de interés en los mercados financieros internacionales, o bien, en otros, la transformación de dicha carga en un subsidio.

\section{El ajuste económico y el mercado de trabajo}

Se argumenta en forma cada vez más frecuente que el aumento de los salarios reales es uno de los factores determinantes principales del crecimiento de la desocupación abierta. Dicha argumentación, cuya base teórica es muy cuestionable dados los supuestos que la fundamentan, no lat corrobora la información existente.
Cuando se analiza la variación de los salarios (sean éstos los mínimos urbanos, los de la industria manufacturera o los de la construcción) con relación a los cambios en la desocupación abierta durante dos subperiodos de la década pasada, los resultados muestran con claridad que dicha relación no existe. La información para 12 
países latinoamericanos referida a los subperiodos 1975-1978 y 1978-1981, seleccionados por las diferentes características de la situación inter nacional predominante en ambos, confirma dicho aserto. (Véase el cuadro 3.)

Así, durante el primer subperíodo sólo en un pais (Venezuela) la baja de los salarios mínimos urbanos estuvo acompañada de un descenso en la tasa de desocupación abierta. Pero aun en este caso dicha relación no aparece cuando se considera la variación de los salarios industriales. Más aún, tampoco hay casos de países donde se observen alzas de salarios reales asociadas con una elevación significativa (mayor que I por ciento) de la desocupación abierta. Por el contrario, en seis de los 12 paises considerados se registra un proceso inverso; es decir, aumentos de los salarios reales acompanados por disminuciones significativas de la desocupación abierta o reducciones de los salarios reales junto con incrementos significativos de la desocupación abierta.

Durante el período 1978-1981 sólo en dos paises se registra simultáneamente una disminu- ción de los salarios reales con reducción de la desocupación abierta, cuando se consideran los salarios mínimos urbanos y los industriales. Asjmismo, al tomar en cuenta también los movimientos del mismo signo, es decir, aumento de los salarios reales e incremento de ta desocupación abierta, el número de casos referidos al salario minimo urbano es de tres, mientras que cuando se consideran los salarios industriales y los de la construcción, no aparece ningún caso significativo. Por otro lado, en un mayor número de países se registran movimientos de signos opuestos entre los salarios reales y la desocupación abierta, cualquiera sea el tipo de salario considerado.

Esta escasa relación entre las variaciones de los salarios reales y los cambios en la desocupación abierta, e incluso el surgimiento de numerosos movimientos de signos contrarios, llevan a investigar la existencia de otros factores causales. En particular, las modificaciones en el nivel de actividad económica pueden generar variaciones en la tasa de desocupación abierta. Ello se des-

Cuadro 3

AMERICA LATINA Y EL CARIBE: SALARIOS REALES, NIVEL DE ACTIVIDAD Y DESOCUPACION ABIERTA

\begin{tabular}{|c|c|c|c|c|c|c|c|c|c|c|}
\hline & \multicolumn{5}{|c|}{$1975-1978$} & \multicolumn{5}{|c|}{ |97k-14⿻4 } \\
\hline & \multirow{2}{*}{$\begin{array}{l}\text { Crecimiento } \\
\text { del pruducto" }\end{array}$} & \multicolumn{3}{|c|}{ Variación de los sadarios" } & \multirow{2}{*}{$\begin{array}{c}\text { Cambios eta la } \\
\text { clesocupación } \\
\text { albicrtat }\end{array}$} & \multirow{2}{*}{$\begin{array}{l}\text { Girecimiento } \\
\text { de! prokluktor" }\end{array}$} & \multicolumn{3}{|c|}{ Variacisin de hes saluriust" } & \multirow{2}{*}{$\begin{array}{l}\text { Cambius en la } \\
\text { descocupación } \\
\text { abierta' }\end{array}$} \\
\hline & & $w_{11}$ & $w_{i}$ & $\mathbf{w}_{\mathbf{c}}$ & & & $n_{m 1}^{+}$ & $n_{1}$ & $w_{\mathrm{b}}$ & \\
\hline Argentina & 1.6 & -50.4 & -54.9 & -55.9 & 0.2 & 0.2 & 8.4 & 19.9 & - & $0.7^{d}$ \\
\hline Brasil & 6.6 & $\$ .5$ & 18.3 & 12.0 & (1).6j & 3.5 & -10.2 & $-6 . .7$ & -15.5 & $1.7^{\circ}$ \\
\hline Cotombia & 6.1 & 9.4 & 13.3 & 5.1 & -2.0 & 4.1 & 211.33 & 7.4 & 14.3 & $-1) .8$ \\
\hline C'osta Rica & 6.9 & 25.3 & 31.3 & 34.9 & 0.4 & 1).6 & 1.8 & 〔).7 & 1.4 & 3.3 \\
\hline Chile & 7.2 & 30.0 & 20.6 & 44.4 & -1.7 & 6.7 & $-1) .6$ & 18.1 & 23.6 & -4.3 \\
\hline Jamaica & -2.1 & $-17.2^{1}$ & & & 4.(1) & $-9.8^{c 1}$ & $-\mid \boldsymbol{2}, 0^{\prime}$ & & & $5.0 \mathbf{r}^{\prime}$ \\
\hline México & 6.6 & 7.9 & 8.0) & - & 0..3 & 8.5 & $-9,0$ & -5.7 & - & -2.4 \\
\hline Panamá & 3.6 & -11.7 & - & -11.7 & 1.0 & 5.6 & 2.1 & - & $-2,5$ & $2.00^{\mathrm{K}}$ \\
\hline Perú & 0.5 & -32.0 & -27.4 & -38.6 & 0.5 & 3.4 & 1.3 .8 & 2.6 & 2.6 & -1.2 \\
\hline Trinidad y Tabago & 9.9 & $8.8^{\prime}$ & & & -1.7 & $4 . y^{\mathrm{kl}}$ & $5 . f^{\mathbf{l}}$ & & & $1.8^{12}$ \\
\hline Urugtay & 4.1 & -16.8 & 26.3 & -25.9 & 1.6 & 4.1 & -15.1 & -15.0 & -19.6 & $-\$ .4$ \\
\hline Venczuela & 6. I & -19.6 & 2.1 & - & -3.2 & -0.1 & $-\mathbf{3 . 0}$ & -12.8 & - & 1.4 \\
\hline
\end{tabular}

Fuence: Vlaborado por PREALC: a basc de infurmación de has palses.

- Tusa anu⿰𠄌三人 de crecintiento.

- Variación porcentual durante el periodo

$w_{10:}$ : salarios minimos urbarnos.

$w_{i}$ : salarios de la industria nanutacturera.

w. isalarios de la censtrucción.

- Puntos parcentuvies entre los arios extremns de la tasa de clesxupación urbina.

" Se refiere a 1978-1980.

- Eutimación preliminar.

1 Se refiere a salarios totales.

* Se refiere * $197 \mathrm{~B}-1979$. 
prende no sólo de una importante corriente de interpretación teórica, sino también porque la información disponible sobre las variaciones a corto plazo parece sugerir la existencia de tal correlación. Al vincular la información disponible sobre crecimiento del producto interno bruto con los cambios en la tasa de desocupación abierta (véase de nuevo el cuadro 3), se observa que durante el período 1975-1978 en cinco de los 12 paises para los cuales se dispone de información se registra una correlación directa entre crecimiento acelerado y disminución de la tasa de desocupación o contracción del producto y au. mento de la tasat de desocupación. Y durante el periodo 1978-1981 nuevamente en cinco de los 12 países considerados se registra la misma relación. Esto indica que existe, a corto plazo, una relacion apreciable entre el nivel de actividad económica y la desocupación abierta, si bien en algunos una tasa de crecimiento elevada aparece asociada a la subsistencia de la tasa de desocupación.

E] análisis precedente permite concluir que ta tasa de desocupación abierta responde más a cambios en el nivel de actividad que a variaciones de los salarios reales. No obstante, es limitada la posibilidad de explicar los cambios en el mercado de trabajo por la mera observación de las variaciones de la desocupación abierta en economias como las de la región caracterizadas por la exis- tencia de magnitudes significativas de subempleo. El ajuste económico en mercados de trabajo heterogéneos puede adoptar diversas variantes, sean éstas cambios en la desocupación abierta, modificaciones en el subempleos o unta combinación de ambos. En lo que sigue se tratará de ilustrar la reacción del mercado de trabajo en cuatro situaciones distintas, de paises latinoamericanos y ante coyunturas recesivas.

Para este efecto, se han seleccionado los casos de Argentina entre 1974-1980; de Chile durante la década pasada; y de Costa Rica y Venezuela a partir de 1978. Los dos primeros, por̈ combinar el ajuste externo con cambios profundos de sus políticas económicas orientadas fundamentalmente a lograr la estabilización de precios y una mayor apertura de sus economias. El tercer pais constituye un caso de economía pequeña y abierta que enfrenta problemas de balanza de pagos y presiones inflacionarias. El último caso ilustra la experiencia de un pás exportador de petróleo que combina la bonanza petrolera reciente con políticas económicas dirigidas a combatir los desequilibrios internos que se manifestaron básicamente por un alza acelerada de los precios comparada con sus patronés históricos.

Como puede observarse en el cuadro 4, la experiencia de los cuatro paises posee caracteristicas comunes. Por un lado, se registran muy moderados ritmos de crecimiento del producto y

Cuadro 4

AMERICA LATINA: TRES TIPOS DE AJUSTE DEL MERCADO DE TRABAJO

\begin{tabular}{|c|c|c|c|c|c|c|c|}
\hline & \multirow{2}{*}{$\begin{array}{c}\text { Crecimiento } \\
\text { anual del } \\
\text { producto } \\
\text { (*) }\end{array}$} & \multirow{2}{*}{$\begin{array}{c}\text { Crecimiento } \\
\text { anual de los } \\
\text { precios } \\
\text { (\%) }\end{array}$} & \multirow{2}{*}{$\begin{array}{c}\text { Variación de } \\
\text { os salarios } \\
\text { reakes entre } \\
\text { extremios } \\
\text { (\%) }\end{array}$} & \multicolumn{2}{|c|}{ Ganiluios entre extremes } & \multirow{2}{*}{$\begin{array}{c}\text { Creximiento } \\
\text { antual de! } \\
\text { HEA no } \\
\text { agricala } \\
\text { (O) }\end{array}$} & \multirow{2}{*}{$\begin{array}{l}\text { Relación in- } \\
\text { gresss sector } \\
\text { informal sec- } \\
\text { tor mo- } \\
\text { dernot }\end{array}$} \\
\hline . & & & & 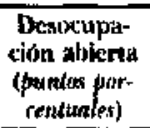 & $\begin{array}{l}\text { Participactión } \\
\text { en el secturs } \\
\text { indormal ur- } \\
\text { bans? }\end{array}$ & & \\
\hline $\begin{array}{l}\text { Caso } 1 \\
\text { Argentina (I974-1980) }\end{array}$ & 1.9 & 156.3 & $-46.9^{\prime \prime}$ & -0.3 & 4.0 & 0.6 & 1,08 \\
\hline $\begin{array}{l}\text { Caso } 2 \\
\text { Costa Rica }(1978-1981) \\
\text { Chile (1970-1980) }\end{array}$ & $\begin{array}{l}0.6 \\
2.7\end{array}$ & $\begin{array}{l}30.1 \\
79.1\end{array}$ & $\begin{array}{l}-11.2 \\
-21.4\end{array}$ & $\begin{array}{r}3.7 \\
14.5^{\prime}\end{array}$ & $\begin{array}{r}-21.1 \\
0.1\end{array}$ & $\begin{array}{l}5.2 \\
2.7\end{array}$ & $\begin{array}{l}0.71 \\
0.73-0.8\end{array}$ \\
\hline $\begin{array}{l}\text { Case } 3 \\
\text { Venezuela (1978-1981) }\end{array}$ & -0.6 & 16.9 & -6.7 & 2.3 & 3.0 & 4.5 & 0.86 \\
\hline
\end{tabular}

Furnte: Elaburado par PREALC; a base de eadatisticas nacionsles.

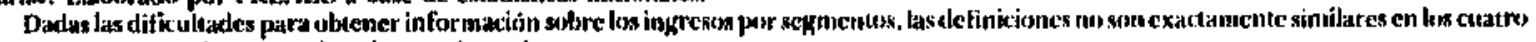
paises peru resultan aproximaciones adecuadas.

Salarios de la jadustria manufacturera.

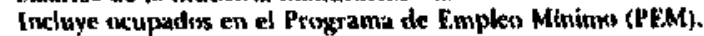


hasta una contracción del mismo en el caso de Venezuela. Por otro, las cuatro economías, aunque en distintos grados, manifiestan problemas inflacionarios. En los casos de Costa Rica y Venezuela, si bien podría considerarse que el aumento de los precios fue relativamente moderado, las tasas de inflación cuadruplican, y hasta quintuplican, las registradas históricamente en estos paises. Por último, durante los perfodos analizados, se registran en todos los casos contracciones de los salarios reales. Estas tres características comunes de hecho expresan decisiones de politicas economicas internas similares destinadas a controlar la demanda $y$, sobre todo, los salarios, con el propósito de reducir el crecimiento de los precios. ${ }^{6}$

La respuesta del mercado de trabajo es, sin embargo, diferente en cada caso. En Argentina, el ajuste se produce por un aumento de la participación de los ocupados en el sector informal urbano, mientras que la desocupación abierta se mantiene virtualmente constante durante el período. En Chile y en Costa Rica, por el contrario, el ajuste se produce casi enteramente por el aumento de la tasa de desocupación abierta, mientras que la participación del sector informal urbano se mantiene constante. Por último, en Venezuela, el mercado de trabajo se ajusta de una manera intermedia, pues se eleva tanto la tasa de desocupación abierta como la participación de los ocupados en el sector informal urbano. En el caso de Argentina el ajuste se efectúa en su totalidad a través del aumento del subempleo; en los casos de Chile y Costa Rica, por elevación del clesempleo abierto; y en el de Venezuela, se distribuye el ajuste entre la desocupación abierta y el subempleo.

Llegado a este punto parecerfa pertinente preguntarse qué hace que el ajuste se efectúe de una u otra manera. Dada la semejanza existente entre los cuatro paises analizados en cuanto a la magnitud de su sector informal, la diferencia

\footnotetext{
'Sabe olservar que la capacialad explicativa de los cambios en los salarios reales $y$ en el nivel de actividiad pari estudiar las variaciones de la desocupacion ibbierta es sumilmente limitada y, muchas veces, contradictoria. Asi, a pesar de que los salarios reales disminuyen en los cuatro costs, lit tasi cle desocupación se mantiene ightal o atmenta en todes les casos.
}

podria encontrarse en el nivel de ingreso medio alli predominante. Asi, tanto en Argentina como en Venezuela los ingresos percibidos por los trabajadores del sector informal presentan diferencias muy reducidas con los ingresos de los sectores modernos. En los casos de Chile y Costa Rica las diferencias alcanzan al 30 por ciento. Ello implicaría que quienes no encuentran trabajo en los sectores modernos, como consecuencia del ajuste que experimenta el mercado de trabajo, prefieren mantenerse en la búsqueda activa cuando las demás oportunidades de ocupación suponen una reducción considerable del ingreso percibido. Y, por el contrario, cuando las diferencias no son tan significativas la opción de ocuparse en el sector informal hasta que el mercado de trabajo se normalice parece constituir una alternativa válida.

Por último, resulta interesante comparar el ajuste del mercado de trabajo en países en desarrollo como los antes analizados, con el efectuado en un pais desarrollado, por ejemplo los Estados Unidos durante el mismo periodo. Según la información disponible para este pais, la poblatción no agrícola creció 2.8 por ciento por año entre 1978 y 1981 , el número de desempleados aument 610.5 por ciento por año y los trabajadores por cuenta propia no profesionales y los familiares no remunerados disminuyeron 6.3 por ciento por año. Este es, claramente, un caso de ajuste a través de incrementos en la tasa de desempleo abierto. Si las diferencias de ingreso en los Estados Unidos son pequeñas, y de todas maneras menores que las prevalecientes en Argentina y Venezuela, debería esperarse una expansión de las ocupacionés no asalariadas, pero la diferencia radica en la existencia de un seguro al desempleo, que en el caso de los Estados Unidos cubre más del 40 por ciento de los desempleados. Para este grupo de poblacion, el desempleo no significa ingresos nulos, sino, por el contrario, un ingreso garantizado mientras continúan buscando activamente nuevos puestos de trabajo.

Además de ilustrar las diferencias que se registran en el tipo de ajuste del mercado de trabajo, el análisis comparativo realizado sugiere que los efectos distributivos son en cada caso distintos. La existencia de un seguro de desempleo, con amplia cobertura a niveles de ingresos normales, minimiza el efecto directo del ajuste en la distribución del ingreso. En el otro extremo, el 
aumento del número de desempleados sin ninguna ayuda compensatoría por parte del Estado, como ocurre en Chile y Costa Rica, deteriora mucho la distribución del ingreso. Los casos intermedios de ajuste por la expansión de las ocupaciones menos remuneradas también afectan negativamente la distribución del ingreso, aun- que de manera menos pronunciada. En este último caso, Ja reducción del ingreso medio de quienes están en ocupaciones por cuenta propia desempeña un papel de seguro colectivo frente al desempleo, y constituye en la práctica una solución subóptima desde el punto de vista del bienestar. 